\title{
Accuracy of critical loads given by substitute sway frames with cladding
}

\author{
F. W. WILLIAMS
}

\section{Dr J. M. Davies, University of Salford}

In reference 2 I proposed an alternative approach to the calculation of approximate elastic critical loads of complex frames based on the same simple substitute frame as that investigated by the Author. This approach involved applying Horne's fictitious side loads ${ }^{8}$ in a sidesway analysis of the substitute frame and then obtaining the critical load from the sidesway deflexions in the manner advocated by Horne. With this method the necessary calculations can be carried out rapidly and manually using Naylor's no shear method of moment distribution. Results similar to those given by the full Horne method were obtained for 15 clad and unclad frames, although with a greatly reduced amount of computation. The method is particularly easy to program for a desk-top computer.

27. In his remarks on reference 2 , the Author fails to comment on its raison d'être. His criticisms are directed towards a secondary aspect. He is correct in his assertion that the figures in table 1 of reference 2 denoted 'Full Grinter' contain systematic errors. These arose as a consequence of a fault in the computer program used to obtain the results and are present only in the case of the more complex frames 7-15. This corrected table is shown as Table 2. It gives rise to conclusions regarding the accuracy of critical loads based on the substitute frame that are identical with those of the Author. Further, my analysis of the frame used as a spot check by the Author (frame 10) used different loads from those used by the Author so that, although the results in reference 2 were internally consistent, the two sets of results were not directly comparable. The values given in Table 2 for frame 10 may be directly compared with those given in the Paper.

\section{Dr R. H. Wood, M}

The Paper confirms conclusively that substitute frames ${ }^{3}$ are powerful, quick to use and sufficiently accurate for designers. However, in examining false results, and criticisms, of Davies ${ }^{2}$ other issues have arisen: what rapid methods exist for designers, how they are designated and what has been accepted in the latest codes of practice. To produce an entirely graphical rapid method, reference 3 proposes using Scott's modified distribution coefficient $k$ for the substitute frame

Paper published: Proc. Instn Civ. Engrs, Part 2, 1979, 67, Dec., 943-949. 


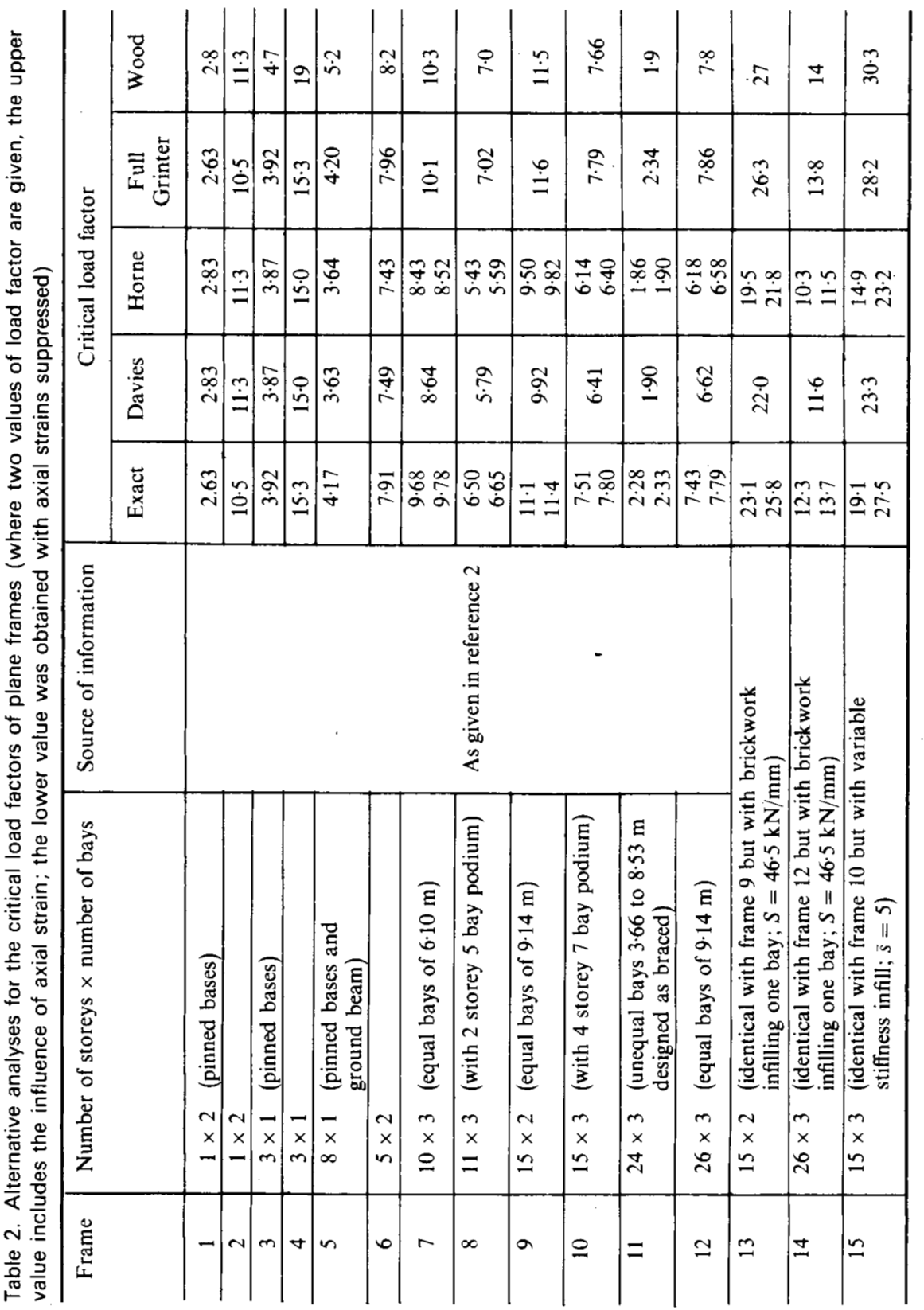


and the optional formula

$$
\lambda_{\mathrm{s}}=\frac{\text { lowest } P_{\mathrm{C}} / P_{\mathrm{E}} \text { in critical storey }}{\text { average } P / P_{\mathrm{E}} \text { at } \lambda=1 \text { of that storey and adjacent storeys }}
$$

29. This method - one of many alternatives - appears to be what Davies ${ }^{2}$ called Wood's method, which was in fact for simple design only. Not until rigid-frame design appeared in 1979 was any mention made of substitute frames, and then there was no mention of equation (1). The 1979 draft BS code of practice has therefore of necessity followed reference 6 , which contained extensive sections based on my substitute frame and cladding treatment, and in particular a revised formula of the type

$$
\lambda_{\mathrm{s}}=\lambda_{\mathrm{c}}\left[\frac{1+\alpha_{u}+\alpha_{1}}{1+\alpha_{u}\left(\lambda_{\mathrm{c}} / \lambda_{\mathrm{u}}\right)+\alpha_{1}\left(\lambda_{\mathrm{c}} / \lambda_{1}\right)}\right]
$$

where

$$
\begin{aligned}
& \alpha_{\mathrm{u}}=k_{\mathrm{u}}\left(\frac{K_{\mathrm{u}}+K_{\mathrm{c}}}{2 K_{\mathrm{c}}}\right) \\
& \alpha_{\mathrm{l}}=k_{1}\left(\frac{K_{1}+K_{\mathrm{c}}}{2 K_{\mathrm{c}}}\right)
\end{aligned}
$$

and the subscripts $\mathrm{c}$, $\mathrm{u}$ and 1 denote critical storey, upper adjacent storey and adjacent storey respectively.

30. Equation (1) was withdrawn because infinitely stiff beams can seal off the effects of adjacent storeys. Equation (2) takes account of

(a) infinitely stiff beams $(k=0)$

(b) whenever $\lambda_{\mathrm{u}}=\lambda_{\mathrm{I}}=\lambda_{\mathrm{c}}$, such coincidence makes $\lambda_{\mathrm{s}} \simeq \lambda_{\mathrm{c}}$

(c) column sections run through would make $\lambda_{u}>\lambda_{c}$, and the effect of $\lambda_{v}$ would diminish

(d) local $\lambda_{\mathrm{c}}$ values are favoured where local column stiffnesses $K_{\mathrm{c}}$ are relatively large.

31. Taking Davies' three-storey frame $\mathrm{e}^{2}$, which fared the worst, the local $\lambda_{\mathrm{c}}$ values are 14.1 (bottom), 19.8 and 51.2 (top). Equation (1) gives 17.9 (cf Davies' value of 19) and equation (2) gives $15 \cdot 3$, which happens to be exact. Williams ${ }^{15}$ has proved that the lowest $\lambda_{\mathrm{c}}$ for any storey, with any ratio $k$, must be a lower bound. In view of the tiny effect on the Merchant-Rankine formula, this lower bound graphical process is the most rapid safe method. It can be checked by graphical or numerical stiffness distribution-local or complete -of the Grinter frame. All these methods are my methods, rather than the one designated by Davies.

32. An attractive feature of graphical stiffness distribution is that the process always stops before an effective column stiffness of $-\infty$ is reached, and the fundamental $\lambda_{\text {s }}$ is guaranteed. The necessary checks using the Author's computer method should be studied; the corresponding check with stiffness distribution 
on desk computers (which do not stop the process) is to count the number of times complete distribution passes through this singularity, which number is the harmonic number for $\lambda_{\mathrm{s}}$-zero if the fundamental $\lambda_{\mathrm{s}}$ is found.

33. Codes of practice demand sway within $1 / 300$ per storey. It is surprising how often this rules out plastic design. Littlewoods' frame was competitively elastically designed, yet would not pass this deflexion test. ${ }^{11}$ The Davies-Horne frames 3,5 and 11 would likewise fail this test, as well as being disallowed for manual plastic design. A halt should be called to further discussion on critical loads, and more energies spent on finding $\bar{s}$ for tests on cladding, for the present safety factors $\left(20\right.$ at $\lambda=1,80$ at $\lambda_{\mathrm{f}}$ ) swamp all other considerations.

\section{Professor Williams}

The confirmation of the conclusions in the Paper afforded by Dr Davies' corrected 'Full Grinter' (i.e. $\lambda_{s}$ ) values of Table 2 is valuable because his problems are more realistic than those of Table 1 , which were chosen to combine "reasonable coverage and concise presentation' $(\S 3)$. However, Dr Wood's comments in $\$ 28-31$ are relevant to the Wood results of Table 2 because, except for frames 10 and 15, these results duplicate the Wood results of table 1 of reference 2, on which Dr Wood's discussion is based.

35. The closeness of the $\lambda_{\mathrm{s}}$ and $\lambda_{\mathrm{c}}$ results in Table 1 and the corresponding closeness of the 'Exact' and 'Full Grinter' results of Table 2 justifies the use of any of the relatively simple methods for finding $\lambda_{\mathrm{s}}$ (i.e. the exact lowest critical load factor of the substitute frame) instead of $\lambda_{\mathrm{c}}$. This closeness is also welcome because otherwise doubt would be cast on the appropriateness of the substitute frame and hence on the validity of any method, including the attractively simple methods of Dr Davies $(\S 26)$ and Dr Wood ( $\$$ 28-31), which replaces $\lambda_{c}$ by an approximation to the substitute frame's lowest critical load factor, i.e. by an approximation to $\lambda_{s}$.

36. Dr Davies and Dr Wood have referred to various methods for finding $\lambda_{s}$, or approximations to it. When a suitable programmable calculator is available it is so easy to find $\lambda_{s}$ that the extra simplicity of methods which find only approximations to $\lambda_{s}$ hardly seems worthwhile. I favour the stiffness matrix method for finding $\lambda_{s}$ given in reference 1 because much of the resulting program and data preparation can be shared with the wind deflexion calculations, ${ }^{1}$ the method is particularly concise, the use of stiffness matrices is widely understood and - of crucial importance - there is no possibility of confusing higher critical load factors of the substitute frame with $\lambda_{s}$. Stiffness distribution is not suitable for programming in its graphical form and its numerical form needs to be handled carefully to eliminate possible confusion between $\lambda_{s}$ and higher critical load factors $(\S 32)$, by using methods which have not been published in detail. ${ }^{9}$

37. When calculations must be performed by hand $\lambda_{3}$ can still be found, ${ }^{1}$ but then the time savings given by the simpler methods which find approximations to $\lambda_{\mathrm{s}}$ are very attractive. Dr Davies' method $(\$ 26)$ has the major attraction of only needing calculations like those needed anyway to find the lateral deflexions due to wind. This is particularly useful if the stiffness matrix method of reference 1 is used because the wind load and the fictitious side loads of $\$ 26$ can be used to generate alternative right-hand sides for equation (19) of reference 1 without duplicating the left-hand side operations, thus making the savings usually made when using the stiffness matrix method with multiple load conditions. 
However, Dr Wood's storey by storey methods ( $\$ \$ 28-31)$ are extremely attractive because they give a valuable insight into the frame's behaviour and allow rapid handling of changes to the design of individual storeys.

\section{Reference}

15. Williams F. W. Simple design procedures for unbraced multi-storey frames. Proc. Instn Civ. Engrs, Part 2, 1977, 63, June, 475-479. 